\title{
O gênero Lentinus Fr. (Tricholomata- ceae) no Estado do Rio de Janeiro.
}

Fátima Maria Amaral Barbosa ${ }^{~}$

1 Bióloga, estagiária do Jardim Botânico do Rio de Janeiro e bolsista do CNPq/ FINEP.

Agradecimentos

A autora agradece ao CNPq/FINEP, pela bolsa concedida e aos pesquisadores Elsie F. Guimarães, Abigail F.

R. de Souza, Honório da Costa M. Neto e Verlande D. Silveira.

Lentinus crinitus (L. ex Fr.) Fr., Lentinus volutinus Fr., Lentinus velutinus var. Lepriourii (Mont.) Dennis e Lentinus nigro-osseus Pilát são relatados para o Estado do Rio de Janeiro, Brasil.

\section{Introdução}

Este trabalho tem como objetivo contribuir para a Taxonomia de Fungos do Estado do Rio de Janeiro. Inicialmente são descritas quatro espécies do gênero Lentinus Fr. que de acordo com a nova sistemática proposta por Ainsworth, Sparrow \& Sussman (1973) pertence à família Tricholomataceae. Entretanto, vários autores (Clements \& Shear, 1931; Teixeira, 1946; Silveira, 1981) consideram este gênero pertencente à família Agaricaceae.

Sua importância, no contexto econômico, deve-se ao fato de sendo destruidores de madeira, acarretam prejuízos comerciais em conseqüência da comprovada utilização em grande escala das madeiras em diversos setores.

Estes macromicetos destroem a madeira através do micélio que cresce em seu interior e, por intermédio da ação de enzimas, transformam-nas em alimentos causando, conseqüentemente, lesões que levam a madeira ao estado de decomposição (Cavalcanti, 1975).

Destaca-se o emprego das espécies de Lentinus crinitus (L. ex. Fr.) Fr. e Lentinus velutinus $\mathrm{Fr}$., na alimentação pela família de índios Sanamás da tribo Yanomamus, segundo Fidalgo \& Prance (1976).

\section{Material e métodos}

As espécies estudadas neste trabalho foram coletadas em diversas regiões do Estado do Rio de Janeiro, incluindo-se, também, neste estudo, os exemplares existentes na Micoteca do Jardim Botânico do Rio de Janeiro.

Nos estudos microscópicos foi necessário que as espécies examinadas sofressem cortes longitudinais e transversais, permitindo, com isto, obsenvações morfológicas e anatômicas com o auxílio do microscópio estereoscópico Zeiss.

Os desenhos das estruturas macroscópicas foram elaborados utilizando-se a câmara clara Wild e as microscópicas a câmara clara Zeiss aplicadas ao microscópio Zeiss.

\section{Descrição do gênero}

Lentinus Fr. Syst. Orb. Veg. 77.1825 Basiônimo: Agaricus crinitus L., Sp. Pl. ed. 2.a p. 1644.1763

Tipo: Agaricus crinitus L. ex Fr., Syst. Mycol. 1, 175. 1821

Píleo geralmente infundibuliforme, coriáceo; superfície dorsal com pilosida- 
des de coloração castanho-claro a castanho-escuro; superfície ventral com lamelas decurrentes de bordos serrilhados ou lisos; haste central; contexto creme claro a escuro, formado de hifas hialinas e septadas; cistídios hialinos, clavados, com paredes espessas; basídios hialinos, clavados; basidiosporos elipsóides a cilíndricos, com parede lisa, hialina e fina.

Quando hidratado, possui a propriedade de retornar ao estado natural. Freqüentemente săo encontrados sobre madeira em decomposição.

\section{Descrição das espécies}

Lentinus crinitus (L. ex Fr.) Fr.

Syst. Orb. Veg. 77. 1825

(Fig. 1 - Fot. 1 e 2)

Basiônimo: Agaricus crinitus L., Sp. PI. ed. 2." p. 1644. 1763.

Agaricus crinitus L. ex Fr., Syst. Mycol. 1. 175. 1821.

Tipo leg. D. Rolander, América Meridional. $=$ Lentinus villosus KI., Linn. 8:479. 1833.

Píleo isolado ou em grupo, coriáceorígido, resistente e com aspecto infundibuliforme quando seco (Foto 1 e 2), quando fresco apresenta-se flexível, com pequena depressão central arredondada, 1,3 a $4,5 \mathrm{~cm}$ diâm.; superfície dorsal (Foto 1) recoberta por espessa pilosidade que geralmente dispõe-se em feixes aglomerados, tomando aspecto escamoso, de coloração castanho-claro a castanho-escuro e, ao envelhecer, os pêlos tornam-se mais enegrecidos, tendendo a superfície ficar glabra; margens com tomento mais denso que a regiäo central, quase totalmente involuta (Foto 2) quando seco, sendo que, quando fresco, é horizontalmente expandida, de bordos ligeiramente divididos em pequenos lóbulos lisos; superfície ventral (Foto 2) glabra, com lamelas decurrentes, interconectadas na base formando uma rede, apresentando pequenas glândulas em toda sua extensão, castanho-claro a castanho-escuro, margens finamente serrilhadas; haste de mesma coloração do píleo, central-cilíndrico, levemente sinuosa, possuindo pêlos delgados e escamosos, recobrindo sua superfície, sendo que às vezes tais pêlos são quase inexistentes, as dimensões da haste variam de acordo com o tamanho do píleo, medindo 1,0 a $6,0 \mathrm{~cm}$ alt. e 0,2 a $0,6 \mathrm{~cm}$ diâm.; contexto creme, medindo $0,1 \mathrm{~cm}$ esp.

Habitat - Foi encontrada em sua gran- de maioria sobre troncos em decomposição; sobre tronco de Lecythidaceae em decomposição.

Material examinado - Parque Nacional de Itatiaia - leg. O. Fidalgo \& $\mathrm{K}$. Fidalgo 275, 13/09/55, det. O. Fidalgo \& K. Fidalgo, RB 217089; Parque Nacional de Itatiaia, Picada Massart, Maromba - leg. O. Fidalgo \& K. Fidalgo s/n. ${ }^{\circ}, 17 / 09 / 55$, det. O. Fidalgo \& K. Fidalgo, RB 217095; Jardim Botânico do Rio de Janeiro - leg. O. Fidalgo \& K. Fidalgo s/n. ${ }^{\circ}, 13 / 10 / 55$, det. O. Fidalgo, RB 217087; Itaboraí - leg. A. F. R. de Souza s/n. ${ }^{\circ}, 16 / 08 / 75$, det. A. F. R. de Souza, RB 210437; São Gonçalo leg. A. F. R. de Souza 7, 06/03/77, RB 178104; Jardim Botânico do Rio de Janeiro - leg. A. Delgado s/n. ${ }^{\circ}, 10 / 11 / 77$, RB 185122: Casemiro de Abreu, Vale do Córrego da Luz - leg. C. M. Pape 56 , 03/11/78, RB 188890; Mangue da Coroa Grande - leg. L. Mendonça $s / n .^{\circ}$, 03/10/78, RB 188897; Barra de São João - leg. C. M. Pape 117, 19/02/79, RB 189113; Restinga do Grumari - leg. M. R. O. Figueiredo et al 7, 13/07/79, RB 190751; Magé, Santo Aleixo - leg. C. M. Pape 285, 20/05/79, RB 191301; Pedra de Itaúna - leg. H. Berandi s/n. ${ }^{\circ}, 11 / 08 / 81$, RB 210438; Jardim Botânico do Rio de Janeiro - leg. A. F. R. de Souza s/n. ${ }^{\circ}$, 08/10/82, det. A. F. R. de Souza, RB 210446; Magé - leg. F. M. A. Barbosa 12. 07/11/82, RB 210449; Jardim Botânico do Rio de Janeiro - leg. F. M. A. Barbosa 23 et al. 18/11/82, RB 210642; Magé leg. F. M. A. Barbosa 28, 20/11/82, RB 210801 ; Jardim Botânico do Rio de Janeiro - leg. F. M. A. Barbosa 33, 02/12/82, RB 210839 .

Discussão - Pilát (1936), considera Lentinus crinitus e Lentinus villosus espécies diferentes; Dennis (1950), menciona a grande variação do número e densidade de pêlos em Lentinus crinitus, considerandoos da mesma espécie apesar de haver evidências em chamar a forma lisa de Lentinus crinitus e a com pêlos de Lentinus villosus. Fidalgo (1968) cita Lentinus crinitus como espécie polimorfa.

\section{Lentinus velutinus Fr. \\ Linn. 5:510. 1830 \\ (Fig. 2 - Fot. 3)}

Tipo: leg. Beyrich, Brasil.

Píleo anual (Foto 3), solitário ou em pequenos grupos unidos pelas bases dos estipes; membranoso quando fresco ou hidratado e coriáceo quando seco; circular, infundibuliforme, 1.5 a $9,0 \mathrm{~cm}$ diâm.; superfície dorsal com coloração amarelada a castanho-escuro (cor de couro quando tratado), ligeiramente velutina, tornando-se estriada nos bordos à medida que ocorre queda dos pêlos; margens com densos pêlos que alcançam até $1 \mathrm{~mm}$ compr. e apresentando-se em feixes, involuta, de bordos sinuosos quando seco e planos quando frescos; superfície ventral glabra com lamelas decurrentes, não anastomosadas na base e desaparecendo gradualmente ao longo da haste, de bordos lisos e uniformemente inteiros, haste central, cilíndrica, rígida, marrom clara, reta ou sinuosa, bastante velutina com pêlos castanhos-escuros, os quais podem atingir $1 \mathrm{~mm}$ compr., possuindo de 0.2 a $0,7 \mathrm{~cm}$ diâm. e 1,0 a $5,5 \mathrm{~cm}$ alt.; contexto brańco a creme, medindo aproximadamente $0,5 \mathrm{~cm}$ esp.

Habitat - Espécie disseminada em sua totalidade sobre troncos secos ou em estado de decomposição; sobre tronco de Lecythidaceae em decomposição.

Material examinado - Nova Iguaçu - leg. N. M. F. da Silva s/n. ${ }^{\circ}, 20 / 11 / 75$, RB 217104: Casemiro de Abreu, Córrego da Luz - leg. C. M. Pape 58, 03/11/78, det. A. F. R. de Souza, RB 189106; Conceição de Macabu - leg. C. M. Pape 75, 09/12/78 RB 188907; Restinga do Grumari - leg. N. M. F. da Silva 691, s/data, RB 174309; Magé - leg. F. M. A. Barbosa 13, 07/11/82, RB 210478; Jardim Botânico do Rio de Janeiro - leg. F. M. A. Barbosa 40 et al, 14/12/82, RB 210901.

Lentinus ve/utinus var. leprieurii (Mont.) Dennis

Kew Bull. 5:326. 1950

(Fig. 3 - Fot. 4)

Basiônimo: Lentinus leprieurii Mont., Ann. Sci. Nat. Ser. 4.1. 119. 1854.

Tipo: leg. Dennis 182. 16/10/1949, Trinidad.

Píleo isolado (Foto 4), coriáceo-rígido quando seco e membranoso quando hidratado, circular, profundamente infundibuliforme, 1,2 a 1,7 cm diâm.; superfície dorsal recorberta por pêlos castanhos-escuros; margens com densos pêlos, fortemente involuta; superfície ventral glabra com lamelas decurrentes, pequenas, pouco sinuosas, rígidas, variando entre o castanhoclaro e o castanho-escuro; haste central, cilíndrica, rija quando seca e flexível após hidratação, castanho-clara a castanho-es- 
curo, reta ou sinuosa, com pêlos curtos dispostos em feixes e apresentando aspecto escamoso, 1,5 a $3,0 \mathrm{~cm}$ diâm. e 2,3 a $3,1 \mathrm{~cm}$ alt.; contexto castanho-escuro, aproximadamente $0,3 \mathrm{~cm}$ esp.

Comentário - Conforme descrito por Dennis (1950), foi observado que esta variedade difere da variedade tipo por apresentar lamelas obtusas, mais estreitas e de tamanho mass reduzido, além de outros caracteres externos visíveis. sic̣ão.

Habitat - Sobre tronco em decompo-

Material examinado - Itaboraí - leg. A. F. R. de Souza s/n. ${ }^{\circ}, 24 / 04 / 80$, RB 198616.

Lentinus nigro-osseus Pilát

Ann. Mycol. 34:122. 1936

(Fotos 5 e 6 )

Tipo: no Herb. Mus. Bot. Berol. leg. Duchassaing 49, Guadalupe.

Píleo isolado, convexo, coriáceo, centralmente umbilicado, medindo aproximadamente $2,6 \mathrm{~cm}$ diâm.; superfície dorsal
(Foto 5) lisa, creme, com estrias castanhoescuro nas proximidades do centro; margens onduladas, estriadas, com pêlos curtos castanho-claros, superfície ventral (Foto 6) glabra, lamelas decurrentes aglomeradas, estreitas, claras, com glândulas em sua extensão podendo serem observadas através da lupa; haste central, glabra, castanho-clara apresentando-se mais escura na base, $2,0 \mathrm{~cm}$ alt. e $1,0 \mathrm{~cm}$ diâm.

Observação: Para que não fosse danificada a coleção da Micoteca do Jardim Botânico do Rio de Janeiro devido à existência de apenas um (1) exemplar desta espécie, só foi possivel classificá-la através de sua morfologia externa.

Habitat - Espécie crescendo sobre madeira em decomposição.

Material examinado - Casemiro de Abreu, Córrego da Luz - leg. C. M. Pape 85, 31/12/78, RB 189102.

\section{Referências Bibliográficas}

AINSWORTH, G. C., SPARROW; F. K. \& SUSSMAN. The fungi and advanced treatise. A taxonomic review with keys. Vol. IV B. Academic Press N. York. 504 págs. 1973.

CAVALCANTI, M. A. Estudo da Familia Po. lyporaceae em Pernambuco. 1975. (Tese de Mestrado).

CLEMENTS, F. E. \& SHEAR, C. L. The genera fungi. 496 págs. The $\mathrm{H}$. W. Wilson Co., New York. 1931.

DENNIS, R. W. G. Lentinus in Trinidad. Kew Bulletin. London. 3:321-333. 1950.

FIDALGO, M. E. P. K. Contribution to the funigi of Mato Grosso, Brasil. Rickia, 3:171-219. 1968.

FIDALGO, O. \& PRANCE, G. T. The ethno. mycology of the Sanama Indians. $\mathrm{Mr}$. cologia, N. Y. 68 (1):201-210. 1976.

PILÁT, A. Revision der tropischen Lentinus - Arten aus dem Herbar des Botanis. chen Museums in Berlin - Dahlem. Ann/s. Mycol. Berlin. 34:108-140. 1936.

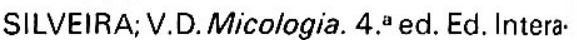
mericana, 1981.

TEIXEIRA, A. R. Himenomicetos brasileiros III (Agaricaceae). Bragantia 6(1):165. 178.1946.

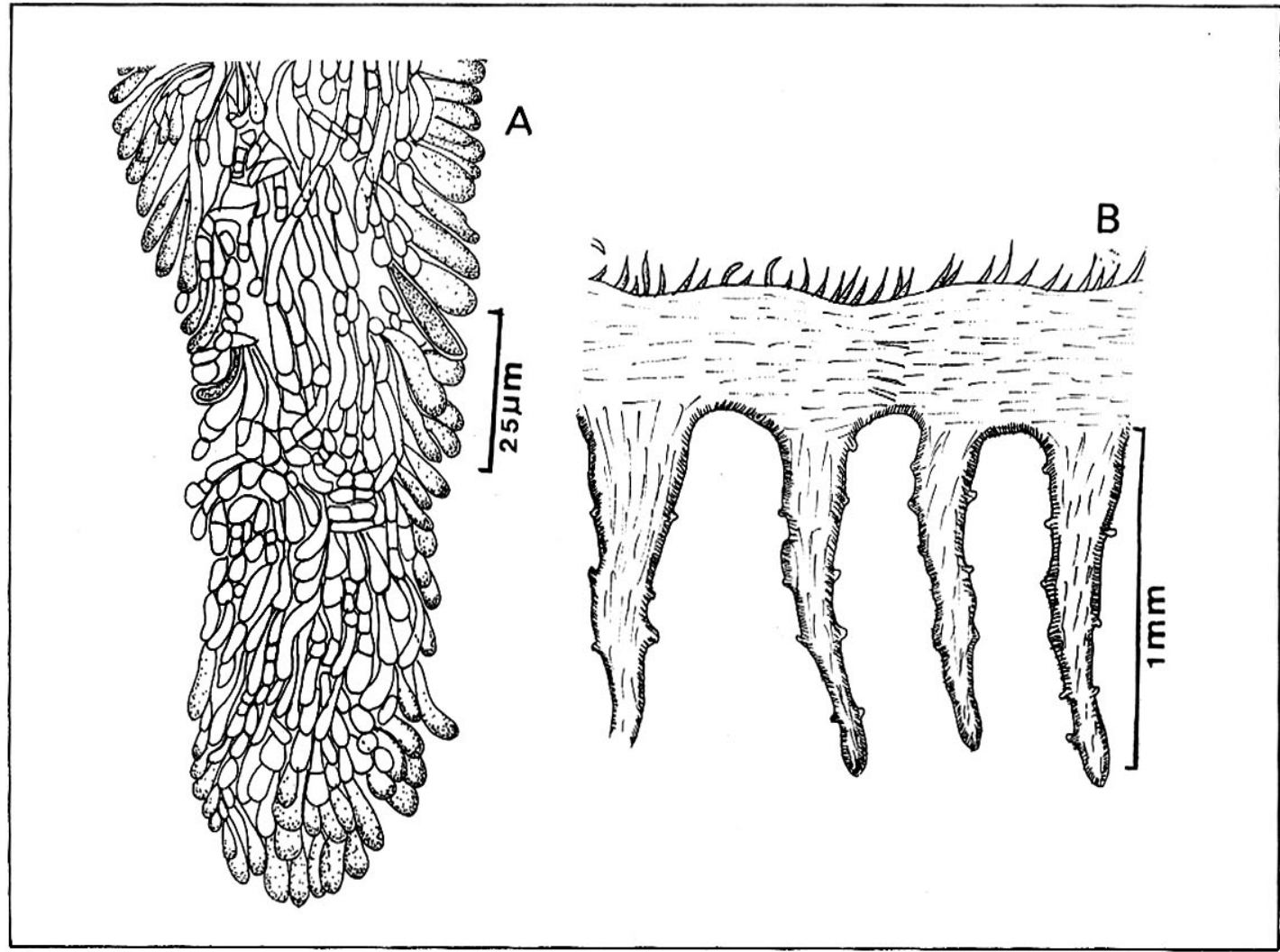

Figura 1

Lentinus crinitus (L. ex $\mathrm{Fr}$.) $\mathrm{Fr}$.

A - Corte transversal do píleo. Extremidade da lamela mostrando as hifas no contexto e a disposição dos basídios.

B - Corte transversal do píleo apresentando as lamelas com glândulas. 


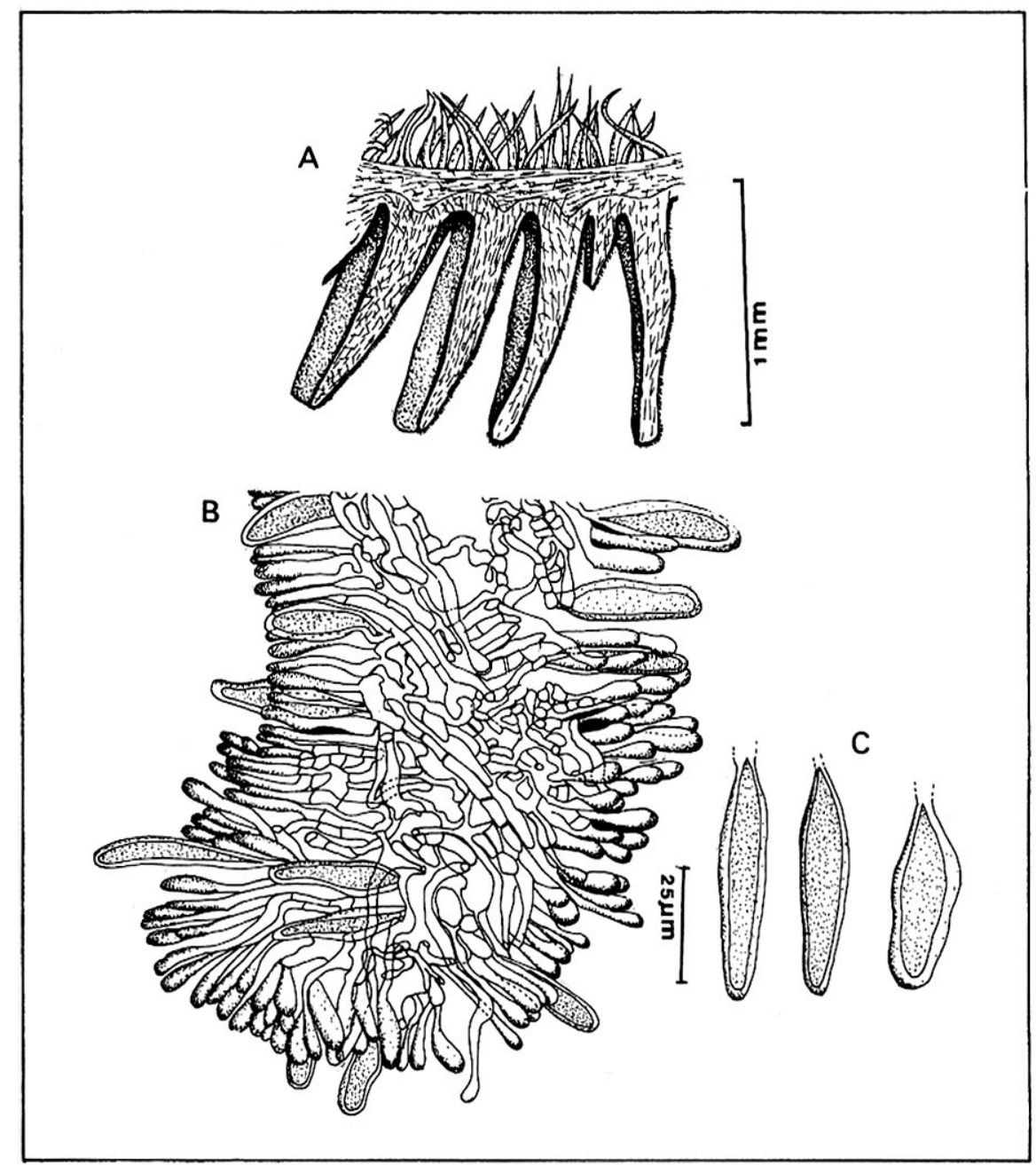

Figura 2

Lentinus velutinus $\mathrm{Fr}$ :

A - Corte transversal do píleo com as lamelas evidenciadas.

B - Corte transversal do píleo. Extremidade da lamela mostrando as hifas no contexto e a disposição dos basídios.

C-Cistídios.

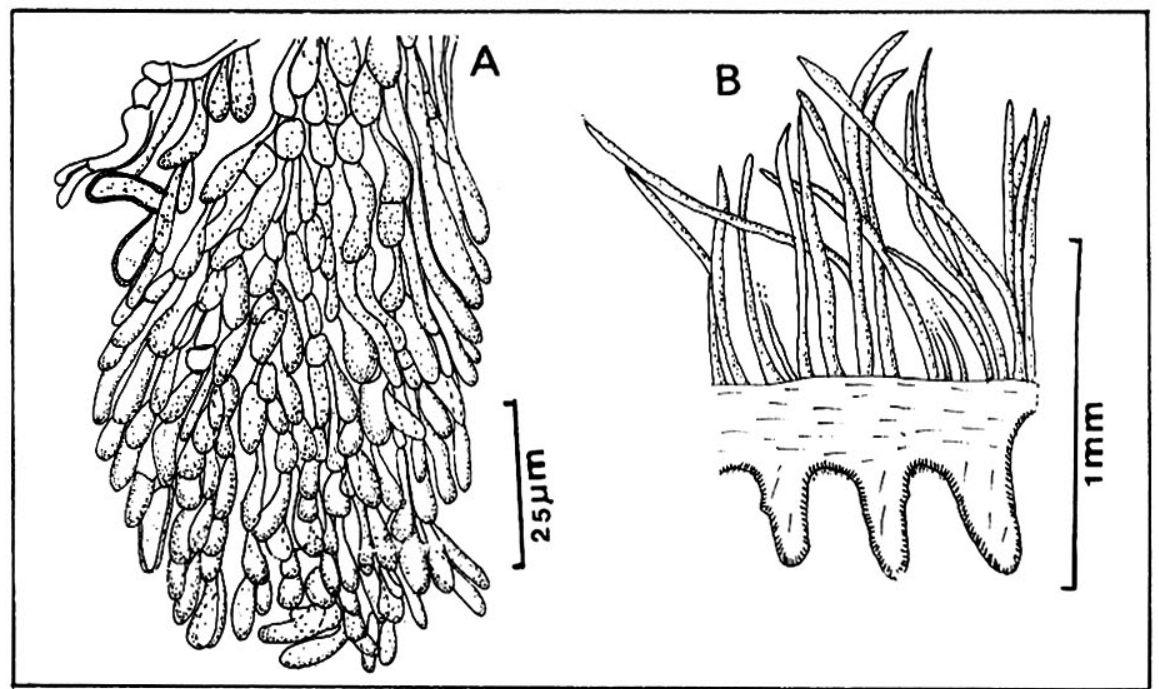

Figura 3

Lentinus ve/utinus var. Ieprieurii (Mont.) Dennis

A - Corte transversal do píleo. Extremidade da lamela mostrando as hifas no contexto e a disposição dos basídios.

B - Corte transversal do píleo apresentando as lamelas. 

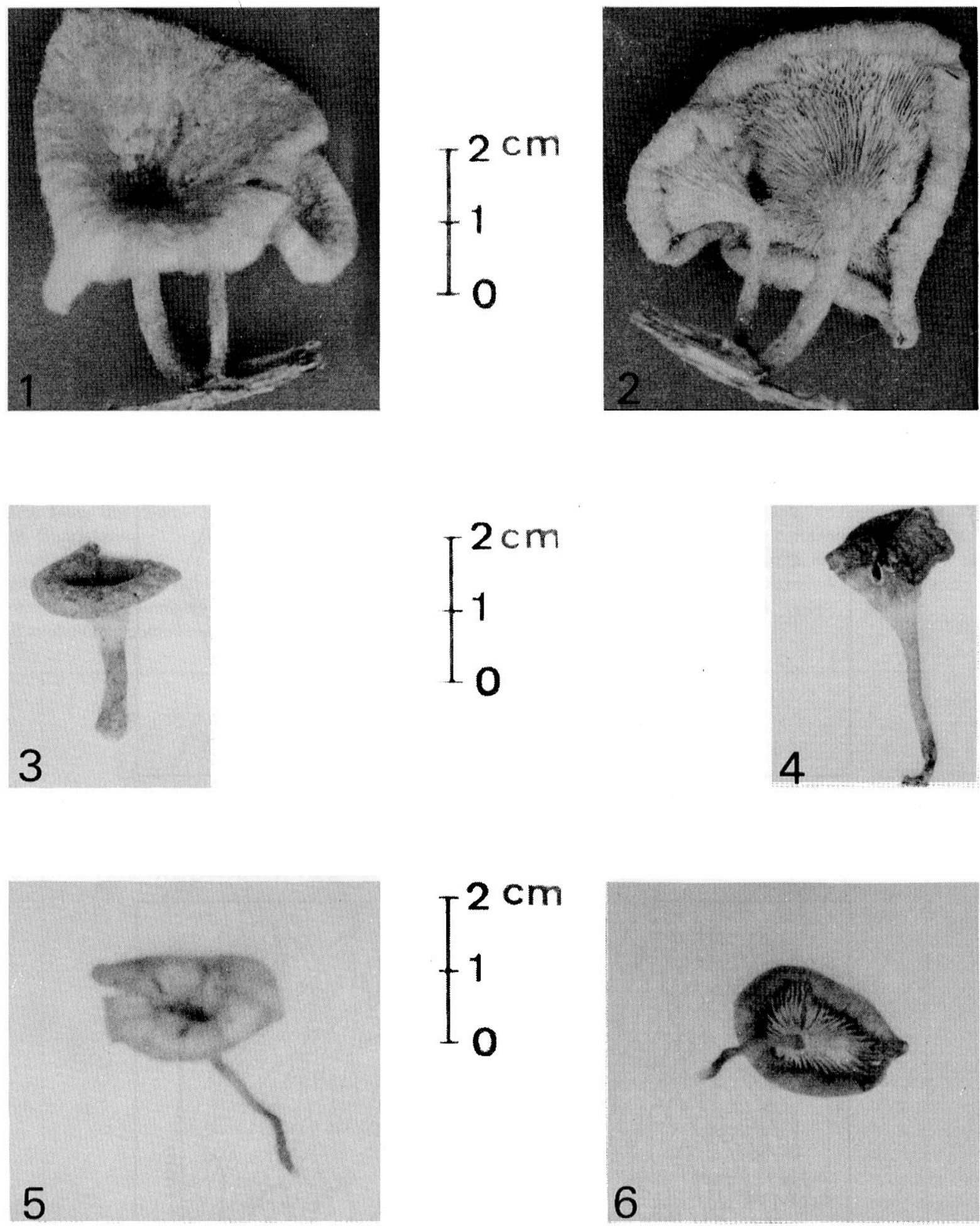

1-2 - Lentinus crinitus (L. ex Fr.) Fr. 1 - Parte dorsal; 2 - Parte ventral; 3 - Lentinus velutinus $\mathrm{Fr} . ; 4$ - Lentinus velutinus var. leprieurii (Mont.) Dennis; 5-6 - Lentinus nigro-osseus Pilát; 5 - Parte dorsal; 6 - Parte ventral. 